\title{
Automated analysis and predictive modeling of craniosynostosis with cranial suture measurements and intracranial volume asymmetries using the snake algorithm
}

\author{
Marcus A Walker ${ }^{1}$, Christopher D Hermann ${ }^{2}$, J oseph K Williams ${ }^{2,}{ }^{3}$, Brani Vidakovic ${ }^{1}$, Rene \\ Olivares-Navarrete ${ }^{4}$, Zvi Schwartz ${ }^{4}$, Barbara D Boyan ${ }^{1,4}$ \\ 1. Georgia Institute of Technology, Institute for Bioengineering and Bioscience, Atlanta, GA, USA. 2. Emory University \\ School of Medicine, Atlanta, GA, USA. 3. Children's Healthcare of Atlanta, Atlanta, GA, USA. 4. School of Engineering, \\ Virginia Commonwealth University, Richmond, VA, USA.
}

Correspondence: Barbara D Boyan. Address: Georgia Institute of Technology, Institute for Bioengineering and Bioscience, Atlanta, GA, USA. Email: bboyan@vcu.edu

Received: February 29, 2016

DOI : $10.5430 /$ jbei.v2n2p132

Accepted: April 19, 2016

URL: http://dx.doi.org/10.5430/jbei.v2n2p132

\section{Abstract}

Quantifying the progression of a condition is one the most difficult challenges facing physicians as they look to provide the most effective treatment customized for individual patients. To achieve these goals, a novel image processing algorithm providing automated quantification has been developed to analyze the cranial vault and sutures using CT in humans and construct predictive models of the condition. Currently, very few preoperative or postoperative quantification tools exist for children with craniosynostosis so we used a customized active contour snake algorithm and 3D reconstruction to quantify the intracranial volume and measure the cranial sutures of these patients.

A set of 117-patient CT scans was collected and analyzed, 77 with varying types of craniosynostosis and 40 normal patients as a control. Total intracranial volume was maintained in craniosynostosis patients in comparison to normal resulting in a uniform growth curve that showed no significant difference between the two groups. Assessing the asymmetry in the intracranial volume and measurements of suture volume and patency resulted in quantitative separations in synostosis types. These separations were further validated by a logistic regression model with pseudo $\mathrm{R}^{2}$ values greater than 0.95 and an $86.9 \%$ average accuracy in cross-validation of the seven groups, which increased to $91.9 \%$ with the removal of the underrepresented lambdoid synostosis group.

\section{Key words}

Craniosynostosis, Predictive modeling, Logistic regression, Snake algorithm, Intracranial volume asymmetry, Cranial sutures

\section{I ntroduction}

Abnormal craniosynostosis is the premature fusion of cranial sutures that occurs in approximately 1 in every 2,000 children affecting cranial vault development and potentially impairing normal neurological function if left untreated ${ }^{[1-4]}$. The premature bone fusion in the developing skull causes restriction and deformation of the calvaria that can result in developmental disabilities, blindness, and even death. Approximately $40 \%$ of craniosynostosis cases are caused by known 
inheritable mutations and are referred to as syndromic forms of craniosynostosis ${ }^{[3,5,6]}$. Syndromic cases are associated with other developmental abnormalities throughout the body and usually result in a fusion of both sides of the coronal suture and may include multiple suture synostosis. The cause of premature bone fusions in the remaining cases is not currently known, but many believe that they are due to an unknown mutation or environmental factors during development. The synostosis type is classified by either the name of the suture that has fused (e.g. metopic synostosis) or the resulting shape of the skull caused by the deformation (e.g. trigonocephaly).

Surgery is typically the only therapy currently available for moderate to severe cases of craniosynostosis. The most common corrective surgery being performed is complex cranial vault reconstruction where the majority of the calvaria is removed. The goal of surgery is to reshape the calvaria with the hope of correcting both the cranial vault asymmetry and in some children, to relieve the increase in intracranial pressure.

While current surgical techniques have minimized complications associated with the initial surgery, a rapid postoperative re-growth of bone at the affected suture, or re-synostosis, has been observed in up to $40 \%$ of patients following surgery ${ }^{[7,8]}$. Re-synostosis is particularly concerning because it often necessitates additional cranial vault reconstruction surgeries, which have been shown to have up to a $13 \%$ chance of potentially fatal complications ${ }^{[9,10]}$. This has been a major consideration because children who have their first surgery at a very young age often require multiple reconstructions before the age of two ${ }^{[7]}$. It has also been observed that infants younger than 6 months at the time of their first surgery are 3 times more likely to develop re-synostosis than children between the ages of 6 months and 2 years old $^{[11]}$.

$\mathrm{X}$-ray computed tomography (CT) imaging of the skull is currently used for preoperative surgical planning and is a useful tool for noninvasively assessing bone formation. By analyzing the CT scans for intracranial volume asymmetries in the calvaria and individual suture developmental abnormalities, a patient can be better evaluated, diagnosed, and treated based on their current situation and disease progression.

Currently, there are very few preoperative quantification tools or postoperative outcome measures for children with craniosynostosis. That is in part due to the difficulty in quantifying the severity and progression of the disease at the time of diagnosis. Intracranial volume has often been studied as a means of measuring the restriction on calvarial development caused by the suture fusion, but the asymmetry in this volume has seldom been quantified and used to assess different variations in the types of synostosis other than observing the deformation. In the past, different formulas were applied to calculate intracranial volume using exterior or radiographic measurements, but these were based off of experimental data that along with a number of simplifying assumptions, were later proved invalid ${ }^{[12]}$. With the development of cross sectional imaging modalities, CT scans have noninvasively provided a more detailed view inside the skull, but often, simplistic approaches were taken to analyze them that do not take into account image reconstruction characteristics for border definitions or a standardization procedure necessary for comparison between patients. While these studies provided a useful baseline for evaluating intracranial volume, only a few evaluate the area of deformation to quantify changes rather than evaluating the volume as a whole ${ }^{[13,14]}$.

To achieve these goals, a novel image processing algorithm has been developed to analyze the cranial vault and sutures using low-dose CT scans to quantify disease severity using predictive modeling. This automated method of evaluating CT scans allows us to quantify cranial development to identify craniosynostosis and understand its progression as a means of assessing the preoperative condition of a patient. This approach is to correct the previous limiting assumptions by using an active contour snake algorithm optimized for bridging gaps and refining borders based on the intensity gradients of medical images ${ }^{[15]}$. This type of algorithm mitigates the effect of quality differences and assumptions present in previous techniques.

A validation of the quantification algorithm measurements can be performed by assessing how well they can effectively predict the type of synostosis of each patient using logistic regression. Logistic regression is a commonly used predictive 
modeling method that uses a linear combination of variables to predict the probability of different outcomes. The weights for each variable in the model are optimized based on a set of training data with known outcomes. Logistic regression is often preferred to other types of regression such as linear regression because the output probability is constrained to values between zero and one ${ }^{[16]}$. To ensure the stability and predictive accuracy of these models, it has been shown that no less than 10 predictors per variable should be used ${ }^{[17]}$. A reduction of the number of variables incorporated is typically done using some standard statistical procedures to compute the most significant contributing variables such as multicollinearity, principal component analysis (PCA), and feature selection algorithms. This type of assessment validates that these methods well describe the prognostic state of a patient and therefore, can be used as a tool in future clinical assessment. A better understanding of the disease and assessment of its current progression can lead to improved patient evaluation, treatment planning, and assessment of surgical techniques.

\section{Materials and methods}

This study was approved by the Georgia Institute of Technology and Children's Healthcare of Atlanta institutional review boards. One hundred and twenty-two preoperative cranial CT scans were obtained from Children's Healthcare of Atlanta at Scottish Rite from 2000 to 2012. Eighty-one of these scans were done on children with craniosynostosis for preoperative surgical planning, and the other forty-one were of children without craniosynostosis and used as the control group. The exclusion criteria for this study were the presence of any metal or high density headwear during the CT scan or too low quality scans defined by a scan slice separation greater than 2.5 millimeters for craniosynostosis scans or 6 millimeters for normal scans. Five patients were excluded from this study; four of which were excluded for not meeting the quality standard and the fifth because of high density material around the head at the time of the CT scan. In an effect to first characterize standard skull deformations, most multiple suture synostosis patients were also not considered in this study as our intent was to establish our methods in cases of single suture synostosis and bicoronal synostosis.

Table 1. Age and weight for craniosynostosis and normal patients

\begin{tabular}{|c|c|c|c|c|c|c|c|}
\hline \multirow{2}{*}{ Group } & \multirow{2}{*}{$\begin{array}{l}\text { Cases } \\
\text { Patients }\end{array}$} & \multicolumn{3}{|c|}{ Age (Months) } & \multicolumn{3}{|c|}{ Weight* (kg) } \\
\hline & & Mean \pm SD & Min & Max & Mean \pm SD & Min & Max \\
\hline Craniosynostosis & 77 & $7.6 \pm 7.4$ & 0.70 & 34.87 & $8.8 \pm 2.2$ & 5.4 & 15.1 \\
\hline Metopic & 20 & $7.0 \pm 5.5$ & 1.40 & 27.03 & $9.0 \pm 2.0$ & 6.2 & 13 \\
\hline Left Coronal & 13 & $9.2 \pm 11.6$ & 1.17 & 34.87 & $8.9 \pm 2.9$ & 5.4 & 15.1 \\
\hline Right Coronal & 9 & $9.6 \pm 8.4$ & 1.17 & 29.23 & $9.7 \pm 2.5$ & 6.6 & 13.1 \\
\hline Bicoronal & 8 & $4.9 \pm 5.1$ & 0.70 & 13.00 & $6.9 \pm 1.3$ & 5.5 & 9.2 \\
\hline Sagittal & 20 & $5.6 \pm 5.7$ & 0.93 & 19.00 & $8.1 \pm 2.0$ & 5.6 & 12.9 \\
\hline Lambdoid & 7 & $12.6 \pm 5.8$ & 3.00 & 18.00 & $10.0 \pm 1.7$ & 7.8 & 13.2 \\
\hline Normal & 40 & $7.7 \pm 4.8$ & 0.93 & 21.00 & $8.4 \pm 2.2$ & 3.8 & 12.7 \\
\hline
\end{tabular}

Note. ${ }^{*}=$ known for 112 of 117 patients. $\mathrm{SD}=$ Standard Deviation. Age and weight are recorded at the time of the operation.

This patient set, after exclusion criteria, was restricted to a maximum of 20 patients from each type of single-suture synostosis (metopic, sagittal, left coronal, right coronal, and bicoronal, and lambdoid) and 40 patients with a "normal" cranial vault control group. These normal patients were children under two years of age who were imaged for head trauma in the emergency department but were found to have no cranial vault fractures or abnormalities. Both groups had similar average ages, weights, and ratios of male to female patients (see Tables 1 and 2). Due to the low incidence of certain synostosis types, 20 patients were not available for all subtypes, but sufficient numbers were present for most types to evaluate differences. 
Table 2. Syndrome, sex and ethnicity for craniosynostosis and normal patients

\begin{tabular}{|c|c|c|c|c|c|c|c|c|c|c|}
\hline \multirow{2}{*}{ Group } & \multicolumn{2}{|l|}{ Cases } & \multicolumn{2}{|c|}{ Sex } & \multicolumn{6}{|c|}{ Ethnicity } \\
\hline & Total & Syn. & $\mathbf{M}$ & $\mathbf{F}$ & White & Black & Hispanic & Asian & Multi-Racial & Not Reported \\
\hline Craniosynostosis & 77 & $9^{*}$ & 43 & 34 & 46 & 13 & 10 & 1 & 1 & 6 \\
\hline Metopic & 20 & 4 & 13 & 7 & 10 & 5 & 0 & 0 & 1 & 4 \\
\hline Left Coronal & 13 & 1 & 7 & 6 & 6 & 3 & 2 & 1 & 0 & 1 \\
\hline Right Coronal & 9 & 1 & 4 & 5 & 6 & 0 & 3 & 0 & 0 & 0 \\
\hline Bicoronal & 8 & $2^{* *}$ & 2 & 6 & 4 & 2 & 2 & 0 & 0 & 0 \\
\hline Sagittal & 20 & 0 & 15 & 5 & 17 & 2 & 0 & 0 & 0 & 1 \\
\hline Lambdoid & 7 & 1 & 2 & 5 & 3 & 1 & 3 & 0 & 0 & 0 \\
\hline Normal & 40 & & 22 & 18 & 33 & 1 & 5 & 0 & 0 & 1 \\
\hline
\end{tabular}

Note. ${ }^{*}=$ known for 76 of 77 patients. $* *=$ known for 7 of 8 patients. Syn. $=$ Syndromic.

Two main types of analysis were performed on the CT scans of each patient for the purpose of synostosis quantification and differentiation. The first type of analysis quantified the intracranial volume asymmetries by dividing the total intracranial volume into four quadrants and then comparing the volume ratios. The second type of analysis quantified the current state of the cranial sutures (fused, partially fused, or patent) using reconstructed images along the suture for which to measure.

\subsection{User interfaces}

Graphical user interfaces were developed to collect information as a starting point for the quantification program. These interfaces were used to determine an initial, approximate global threshold for bone attenuation in each CT scan and specify anatomical locations on the skull using two-dimensional (2D) cross sectional image slices and three-dimensional (3D) renderings of the skull (see Figure 1A). These anatomical locations include a set of points along the path of each suture as well as the start of the metopic suture, end of the sagittal suture, and farthest ends of the coronal suture visible from a top down view of the skull. These locations were used for the path fitting and dividing the intracranial volume into quadrants along with the upper most point of the petrous portion of the temporal bone underneath the brain that was used as a lower bound for the intracranial volume.

\subsection{Snake border refining}

The bone attenuation threshold was then applied to each 2D CT image slice (see Figure 1B) to create a boundary between areas of higher attenuation (bone) and areas of lower attenuation (everything else). The resulting border on the inside of the skull becomes the initial intracranial volume boundary. The program then refines this border using an active contour snake algorithm previously developed to modify bone borders in medical images by bridging the bone gaps when necessary yet fitting tightly to the bone at the location of the largest intensity drop ${ }^{[15]}$. The previously published program was refined for this specific use by experimentally adjusting the two constant equation parameters as well as the number of harmonics applied to best fit this patient population with wide ranging levels of development. It does this by modifying the initial border to best fit the 2D gradient of the image that identifies the locations where the largest intensity changes occur. This provides a refined inner bone boundary that defines the intracranial volume of the skull (see Figure 1, B-C).

\subsection{Volume asymmetries}

Border refinement was done for each 2D CT slice from upper most point of the petrous portion of the temporal bone underneath the brain to the top of the skull. The volume inside the border for each CT slice in this range was combined together to make up the total intracranial volume. Asymmetries in the intracranial volume were assessed by dividing it into 
four quadrants using two cutting planes. One passes through the midline of the skull, from the start of the metopic suture to the end of the sagittal suture and divides the volume into left and right hemispheres.

The other cuts through the farthest ends of the coronal suture visible from a top down view of the skull and divides the volume into anterior and posterior hemispheres (see Figure 1, D-E). These anatomical locations were selected for each patient using the aforementioned graphical user interfaces for standardization between patients. Measurements were calculated by evaluating the percentage of volume in each quadrant and hemisphere to the total intracranial volume and calculating volume ratios comparing the volume distributions in different areas such as the anterior to posterior ratio.

\subsection{D reconstructions}

After quantifying the volumetric differences, the next task was measuring the cranial suture responses in craniosynostosis. In order to make precise measurements of the developing sutures, a previous methodology was modified from Hermann et al. ${ }^{[15]}$ to create images perpendicular to the skull along each suture path. Perpendicular images are crucial because they visually construct what a perpendicular cut through the skull would look like if done surgically. This was done by using the locations identified along the sutures (see Figure 1F) to create a continuous path for each through the process of 3D polynomial fitting to the typical shape of that suture (see Figure 1G). A 3D surface was then fit to the top of the skull (see Figure 1H) so that at each voxel location along the suture, three vectors can be created: one in the direction of the suture path, one normal to the surface, and one orthogonal to the others. The normal vector and orthogonal vector at each voxel location define a plane, and a $50 \mathrm{~mm} \times 50 \mathrm{~mm}$ reconstructed image was created using 3D linear interpolation (see Figure 1I). For each patient, this process created a stack of $2 \mathrm{D}$ images spanning the distance of each suture that quantify and characterize the suture at that location. Due to the frequency of these planes along the suture path, sets of 100 and 250 reconstructed image planes per suture were constructed.

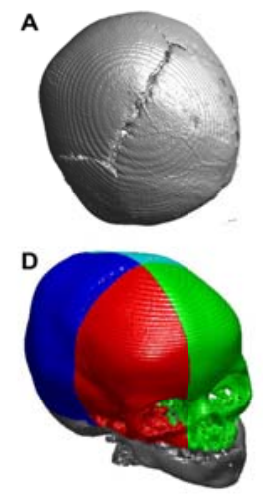

G

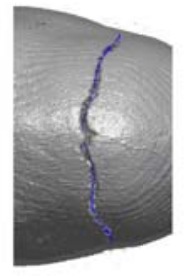

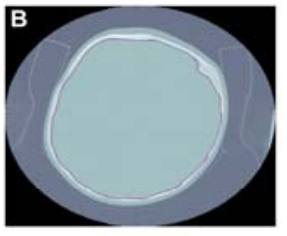

E

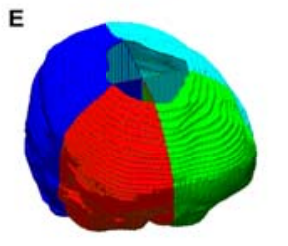

H

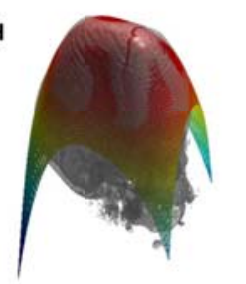

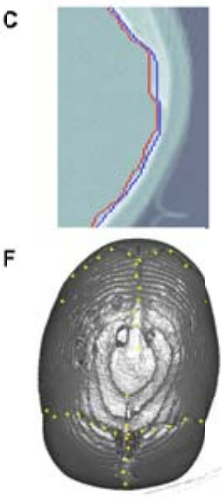

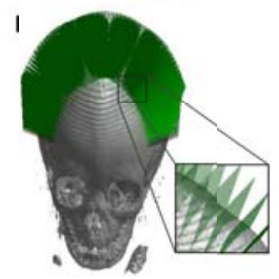

Figure 1. Suture quantification and asymmetry analysis methods. Anatomical locations were selected on a 3D rendering of the skull from a top down viewing angle that allows for a view of the fused and patent sutures (A). The bone border of each CT slice was refined independently starting with the global threshold determined border in red that was fit using the snake algorithm to the exact bone boundary in blue (B-C). The corresponding locations on the skull (D) were used to divide the intracranial volume into four quadrants $(\mathrm{E})$. The suture measurements were calculated by fitting by selecting locations along the suture path marked in yellow (F) and fitting a curve to these points in blue (G). Lastly, a 3D surface was fit over the calvaria $(\mathrm{H})$, and hundreds of image planes were created orthogonal to the surface along each suture path (I). 


\subsection{Using the reconstructions}

An initial bone border was then determined for each image in the stack using a global threshold, and the snake algorithm refined each of these to an exact border ${ }^{[15]}$. Five parameters were measured from the bone borders in each of these images based on previous suture measurements from CT images ${ }^{[18]}: 1$ ) tangent distance, which is the distance from the closest point on the left bone to the closest point on right bone; 2) horizontal distance, which is the shortest distance between two edges of bone directly opposite one another; 3 ) bone height, which is the average height of the bone at all locations where bone is present in a $10 \mathrm{~mm} \times 10 \mathrm{~mm}$ region centered on the suture path; 4) bone volume, which is the amount of bone in a $10 \mathrm{~mm} \times 10 \mathrm{~mm}$ range around the suture path; and 5) openness, which is the determination if the suture is opened (1) or closed (0) based on the number of distinct bone pieces detected in the original $50 \mathrm{~mm} \times 50 \mathrm{~mm}$ reconstructed image.

The distance measurements were recorded as zero if only one bone was detected in a case where the suture was fused. The reduction to analyzing $10 \mathrm{~mm} \times 10 \mathrm{~mm}$ images for bone height and volume was done to analyze only the bone right at the suture interface, not including surrounding bone densities.

\subsection{Fontanel correction}

Due to rapid calvarial development in the first two years after birth, the size of the anterior fontanel varies dramatically based on the patient's age since it is the location where multiple sutures meet on the top of the skull. It has typically been observed to close between the first 7 to 19 months in comparison to the posterior fontanel, which typically fuses the first 1 to 2 months ${ }^{[19,20]}$. A large age-dependent affect like this is often a major source of error. To compensate for these effects, each suture (except for the lambdoid suture as it does not touch the anterior fontanel) was divided into four sections and only the outer three-fourths that will not be affected by the fontanels were analyzed. This method allowed for unbiased analyzing of the cranial sutures while mitigating fontanel effects. The lambdoid suture does not come in contact with the anterior fontanel but was also reduced to analyzing the middle one-half as that is the main portion of it observed to be affected by cranial abnormalities.

\subsection{Border and measurement validation}

Validation of the algorithm was performed by randomly selecting 12 patients from the different types of synostosis. Three metopic, three sagittal, three coronal (one left coronal, one right coronal, and one bicoronal), and three lambdoid were used. Five reconstructed images were randomly selected along each of the four sutures. This resulted in 20 images per patient for a combined total of 240 images. Manual borders were created by manually outlining the suture boundary by a blinded individual. These images were then analyzed by the algorithm described above. These two borders were then compared by calculating the suture measurements on each. Each measurement was evaluated using individual scatter plots of the manual versus automatic border. A linear regression was calculated for each plot and with the resulting $R^{2}$ value.

\subsection{Logistic regression for predictive validation}

To validate the predictive ability of the measurements from our quantification program, a logistic regression model was implemented to show the accuracy and relative variation for each type of synostosis. This model was created using standard methods of feature selection for increased stability and cross-validation to assess its predictive accuracy. Due to an overlap of measurement information for many types of synostosis, a multinomial logistic regression model was used to characterize the differences. Each binomial regression equation compares an outcome type back to the control measurement; in this way, all groups were compared to the normal patient group. To first eliminate redundant information in the feature set, the presence of multicollinearity was assessed by removing features with large variance inflation factors meaning they provide very little unique information. Backward variable selection was then performed on the principal components of the reduced feature set to maximize the variance captured in each variable with the Nagelkerke Pseudo $\mathrm{R}^{2}$ used as the stopping criterion. These procedures reduced the quantification output measurements down to the 11 principal components most significant for prediction satisfying the one-tenth stability criterion. A 10,000 fold cross-validation was then performed on the model using two-thirds subsets of each synostosis type for training and the remaining one-third for 
validation of the prediction accuracy. The full model was also assessed for its data fitting using the McFadden, McFadden Adjusted, Cox-Snell, and Nagelkerke Pseudo $\mathrm{R}^{2}$ values ${ }^{[16]}$.

\subsection{Development and statistics}

The development of these algorithms was done in MATLAB 7.14 (MathWorks), and all data analysis were performed using Graphpad Prism 5 software (GraphPad Software, La Jolla, CA). All data were calculated as the mean \pm SEM with statistical significance between the groups analyzed by one-way ANOVA and Bonferroni's modification of Student's $t$-test. $P$ values $\leq .05$ were considered significant.

\section{Results}

\subsection{Patient population}

There was no significant difference between the average ages for the craniosynostosis and normal groups, 7.6 and 7.7 months respectively, and the same was true for the weights, 8.8 and 8.4 kilograms respectively (see Table 1). When the synostosis types were broken into groups, there was more variation in the mean, ranging from 4.9 to 12.6 months, but no statistical significance was observed in the age or weight. Nine cases of observed syndromic craniosynostosis were present mostly comprised of four with metopic synostosis and two with bicoronal synostosis but with the majority of patients being nonsyndromic. The male to female ratios of patients with craniosynostosis to those without were similar as well, 1.26 and 1.22 respectively. When the synostosis types were broken into groups, there was more variation in the ratio, ranging from 0.33 to 5 since certain subtypes have been seen more predominantly in one sex. Varying ethnicities were seen from all groups, but patients were predominantly white.

Table 3. Measurement validation results for each suture

\begin{tabular}{|c|c|c|c|c|c|c|c|c|c|c|c|c|}
\hline & \multicolumn{3}{|c|}{ Metopic } & \multicolumn{3}{|c|}{ Sagittal } & \multicolumn{3}{|c|}{ Coronal } & \multicolumn{3}{|c|}{ Lambdoid } \\
\hline & $\mathbf{M}$ & B & $\mathbf{R}^{2}$ & $\mathbf{M}$ & B & $\mathbf{R}^{2}$ & $\mathbf{M}$ & B & $\mathbf{R}^{2}$ & M & B & $\mathbf{R}^{2}$ \\
\hline $\begin{array}{l}\text { Tangent } \\
\text { Distance }\end{array}$ & 0.9447 & 0.3856 & 0.9791 & 0.8933 & 1.849 & 0.3400 & 0.9975 & 0.9688 & 0.7387 & 0.6924 & 0.1965 & 0.7107 \\
\hline $\begin{array}{l}\text { Horizontal } \\
\text { Distance }\end{array}$ & 0.8937 & 0.3099 & 0.9752 & 0.8368 & 1.997 & 0.2720 & 0.9151 & 0.1573 & 0.9100 & 0.5966 & 0.1843 & 0.7414 \\
\hline $\begin{array}{l}\text { Bone } \\
\text { Height }\end{array}$ & 0.1432 & 2.576 & 0.0286 & 0.1536 & 2.412 & 0.0710 & 0.3290 & 1.984 & 0.1678 & 0.6402 & 1.348 & 0.5091 \\
\hline $\begin{array}{l}\text { Bone } \\
\text { Volume }\end{array}$ & 1.015 & 1.105 & 0.6927 & 0.7140 & 3.105 & 0.5972 & 0.7137 & 4.031 & 0.4789 & 0.6859 & 6.115 & 0.4970 \\
\hline $\begin{array}{l}\text { Percent } \\
\text { Open }\end{array}$ & 0.9870 & 2.338 & 0.9796 & 0.4454 & 54.78 & 0.3736 & 0.9045 & 11.6 & 0.8679 & 0.6897 & 18.62 & 0.3550 \\
\hline
\end{tabular}

Note. Coefficients from the equation: $\mathrm{y}=\mathrm{Mx}+\mathrm{B}, \mathrm{M}=$ slope. $\mathrm{B}=\mathrm{y}$-intercept.

\subsection{Validated measurements}

Correlation graphs with linear regressions validated the snake algorithm modified bone boundary by comparing the results of four suture measurements with the manual versus automatic border: tangent distance (see Figure 2, A-D), horizontal distance (see Figure 2, E-H), bone volume (see Figure 2, I-L), and percentage open (see Figure 2, M-P). The bone height measurement graphs are not shown in Figure 2 because a good correlation was not achieved, and this measurement was removed from further analysis. A correlation between the manual and automatic borders is represented by linear regression equations that best fit the measurements taken with a slope and $\mathrm{R}^{2}$ value close to one (see Table 3 ). The measurement validation resulted in $\mathrm{R}^{2}$ values ranging from 0.98 at its best to 0.27 at its worst. The metopic suture had the highest correlation with a range from 0.98 to 0.69 . The sagittal and coronal sutures had ranges of $0.60-0.27$ and $0.91-0.48$ respectively. The lambdoid suture also saw a similar range of $0.74-0.36$. The slopes were also seen to range between 1.02 and 0.14 . 

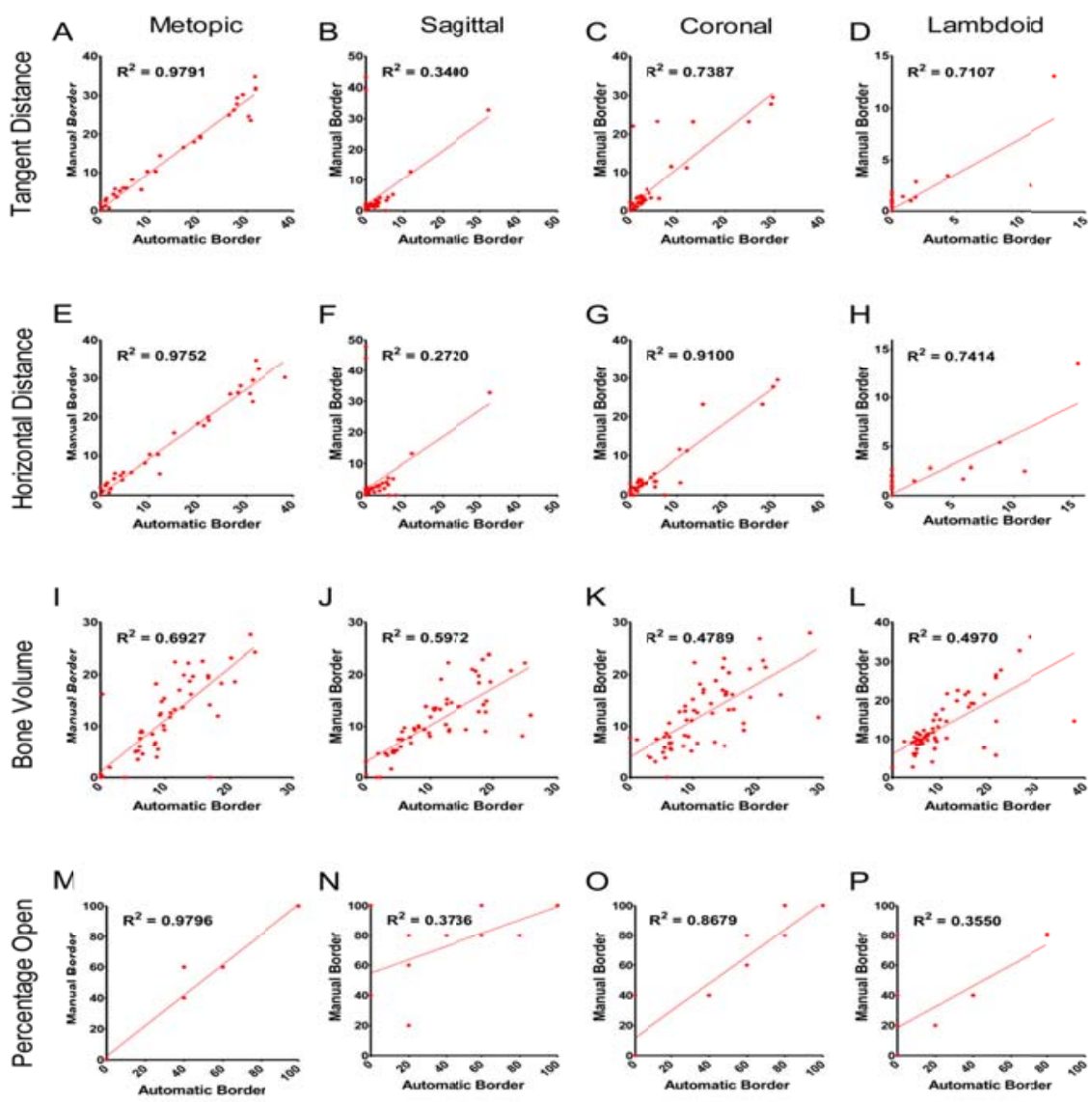

Figure 2. Suture measurement and border validation graphs. A validation of the snake algorithm modified bone boundary was performed with correlation graphs comparing the results of four suture measurements with the manual versus automatic border. The rows contain values for the tangent distance (A-D), horizontal distance (E-H), bone volume (I-L), and percentage open (M-P). The columns correspond to the suture from which the measurements were taken. The calculated $\mathrm{R}^{2}$ values for each linear regression are displayed.

\subsection{Intracranial volume ratios}

A total intracranial volume growth curve (see Figure $3 \mathrm{~A}$ ) was constructed using the full set of patients and resulted in a fairly uniform growth curve with rapid growth within the first 12 months after birth and continued expansion afterward. The growth rate began plateauing around $1,000 \mathrm{~cm}^{3}$ at approximately 10 months after birth. When a non-linear regression fit was calculated for craniosynostosis and normal cases separately, the curve fits were very similar, and the $95 \%$ confidence intervals overlapped with no significant difference (see Figure 3B).

In order to correct for the age dependent variations of the cranial volume measurements, the parameters of interest were normalized by the total intracranial volume. The differences among the synostosis types and normal development were first seen when dividing the intracranial volume into quadrants by splitting it down the suture paths. These differences were first observed from measuring the percentage of the total intracranial volume in the right and left hemispheres (see Figure 3C). In cases of left and right coronal synostosis, the opposite hemisphere was seen to have an increased percentage of the total volume to approximately $60 \%$ with the opposite falling to about $40 \%$, and all other cases had close to $50 \%$ volume in each hemisphere. The anterior to posterior volume ratio showed a separation between sagittal and metopic synostosis at an approximate threshold of 0.23 with $90 \%$ of sagittal synostosis cases above and $90 \%$ of metopic synostosis cases below. 

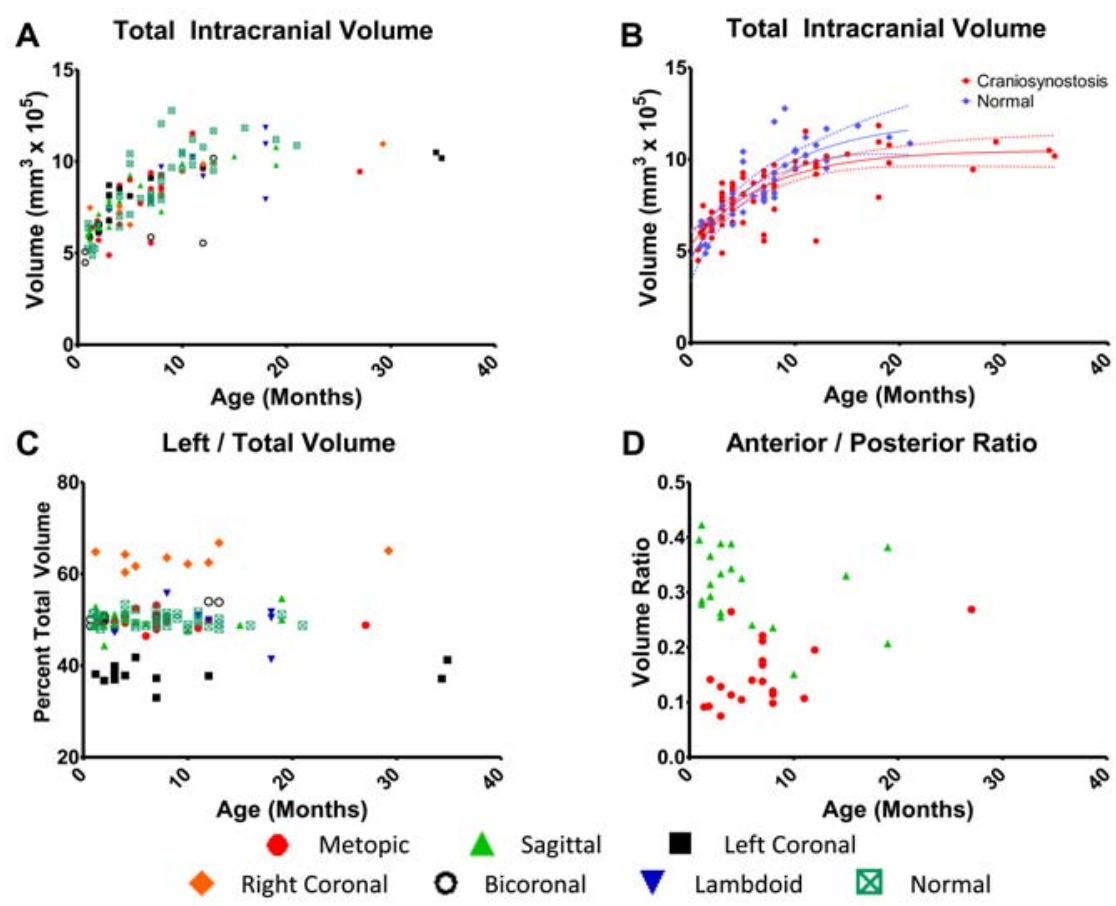

Figure 3. Intracranial volume ratios. The total intracranial volume for all patients was observed to follow a standard growth curve (A). When a non-linear regression fit was calculated for all craniosynostosis and all normal cases, the curve fits were very similar, and the $95 \%$ confidence intervals overlapped with no significant difference seen (B). From these observations, the volume ratios were quantified to calculate the volume displacement. A separation of left and right coronal synostosis, in black and orange respectively, compared to all of the other groups was verified when looking at the percentage of the total volume in the left half (C) and right half of the skull. The anterior to posterior volume ratio also showed a distinct separation between metopic synostosis in red and sagittal synostosis in green (D).

\subsection{Percentage open of each suture}

In addition to analyzing volume ratios, measuring the reconstructed sutures allows for characterization and quantification of their current state. These measurements were assessed for each suture individually with the patients separated into groups by their type of synostosis and the group of normal patients without craniosynostosis. For example, patients with metopic synostosis and therefore, a fused metopic suture were shown to have a negligible percentage of the suture still open (3.4\%), as expected (see Figure 4A). Interestingly, in patients with other fused sutures, the percentage patency of the metopic suture also showed a relative deviation from the measurements of the normal population. This effect was particularly noticeable in patients with bicoronal synostosis where there was a significant increase in the amount of the metopic suture that was open to $71.8 \%$ compared to normal which was only $30.7 \%$.

The percentage open of other sutures followed similar trends. The sagittal suture (see Figure 4B) saw a significant reduction in patency compared to the $76.1 \%$ average of the normal group in cases of sagittal $(21.6 \%)$ and lambdoid synostosis (31.4\%). When assessing both the left and right coronal sutures (see Figure 4, C-D), a fusion of one side of the coronal suture results in a decreased patency to $9.1 \%$ and $12.3 \%$ respectively while most all of the other types did not vary significantly from normal. This reduction was also seen in cases of bicoronal synostosis where that particular suture was fused as well, and patency was measured on average to be $5.8 \%$ on the left side and $6.3 \%$ on the right side. Cases of sagittal synostosis had a slightly elevated patency in the coronal suture to $60.7 \%$ and $57.6 \%$ compared to the normal $40.3 \%$ and $40.7 \%$ due to an elongation and expansion of the front of the skull. The lambdoid suture (see Figure 4E) also followed an analogous trend seeing a significant reduction from normal (39.6\%) only in cases of metopic (8.8\%) and lambdoid (2.9\%) synostosis. 


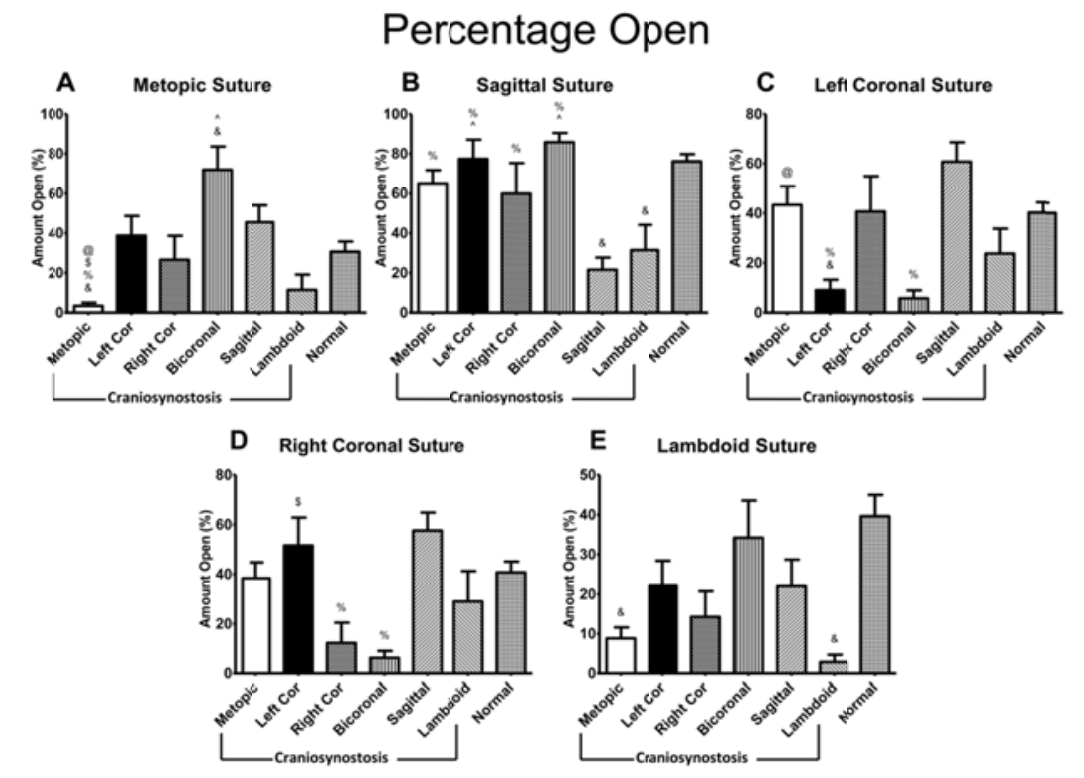

Figure 4. Percentage open suture measurements. The percentage open of the suture was seen to effectively quantify suture fusion and development. In the metopic suture (A), patients with metopic synostosis where seen to have a very low percentage open. This paired affect was seen in each suture as expected, but other types of synostosis were observed to have differences in the sutures that did not have a synostosis. Similar correlated and uncorrelated affects were observed in the other sutures as well (B-E). For all graphs, significance of $P<.05$ for $@$ vs. Left Coronal, \# vs. Right Coronal, \$ vs. Bicoronal, \% vs. Sagittal, ^ vs. Lambdoid, \& vs. Normal.

\section{Bone Volume}
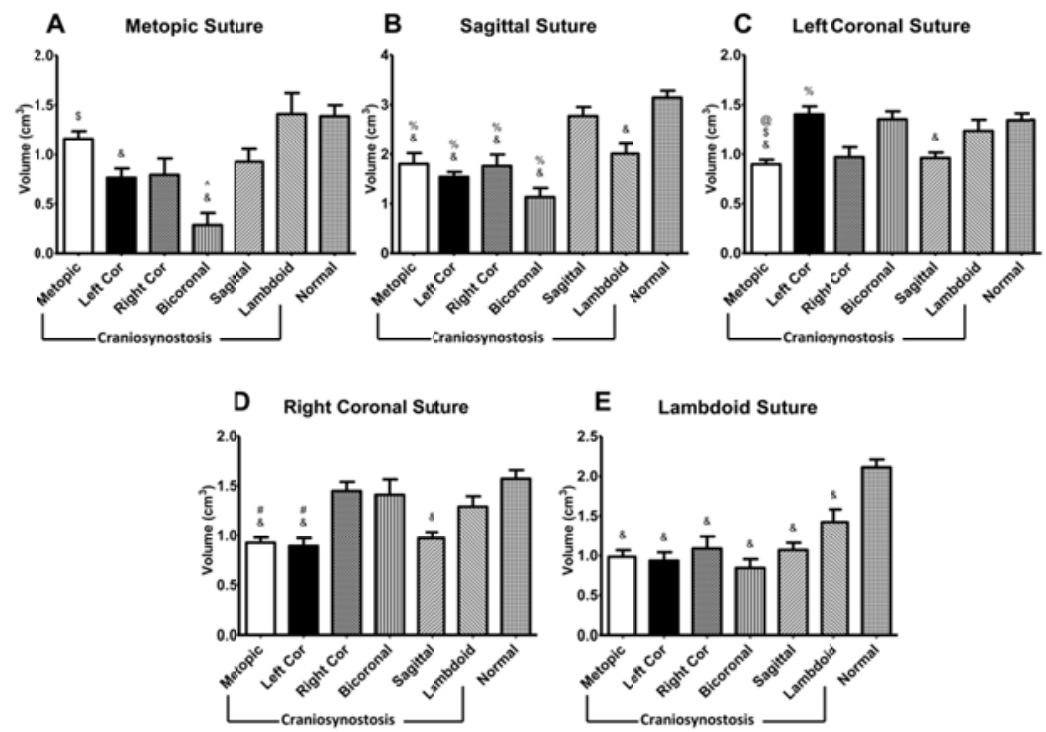

Figure 5. Bone volume suture measurements. The bone volume calculates the amount of bone in a $50 \mathrm{~mm} \times 50 \mathrm{~mm}$ section around the suture path for the entire length of the suture. It was observed to have a near inverse relationship with the percentage open which can be seen in the metopic suture (A) where metopic synostosis patients have an elevated bone volume compared to a decrease in bicoronal synostosis patients. Similar correlated and uncorrelated affects were observed in the other sutures as well (B-E). For all graphs, significance of $P<.05$ for @ vs. Left Coronal, \# vs. Right Coronal, \$ vs. Bicoronal, \% vs. Sagittal, ^ vs. Lambdoid, \& vs. Normal. 


\subsection{Bone volume in each suture}

The bone volume in the metopic suture (see Figure 5A) was at its lowest in cases of bicoronal synostosis $\left(0.286 \mathrm{~cm}^{3}\right)$ as a result of their wider midline of the skull. Metopic synostosis patients had elevated bone volume in the metopic suture of $1.158 \mathrm{~cm}^{3}$ compared to most other types of synostosis, as expected, since the bone had fused together creating more bone along the suture path. Lambdoid synostosis was also seen to have an elevated average volumes of $1.411 \mathrm{~cm}^{3}$, but as previously shown, the average age of these patients was higher (12.6 months) than other types of craniosynostosis. Therefore, many of them had a naturally fused metopic suture.

The sagittal suture (see Figure 5B) produced similar findings to that of the metopic with an increased volume in cases of sagittal synostosis to $2.772 \mathrm{~cm}^{3}$ in comparison to the other types of craniosynostosis. The bone volume in the left and right coronal sutures (see Figure 5, C-D) showed increased volume in all cases of coronal synostosis compared to the rest. An assessment of the lambdoid suture volume (see Figure 5E) yielded a slight increase in volume of patients with lambdoid synostosis to $1.422 \mathrm{~cm}^{3}$ over other craniosynostosis cases.

\section{Metopic Suture Percentage Open \\ Segments $1-4$}
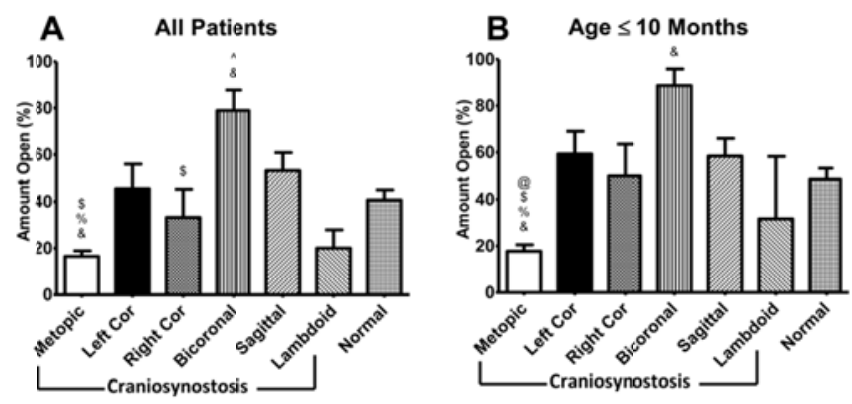

Segments 1-3
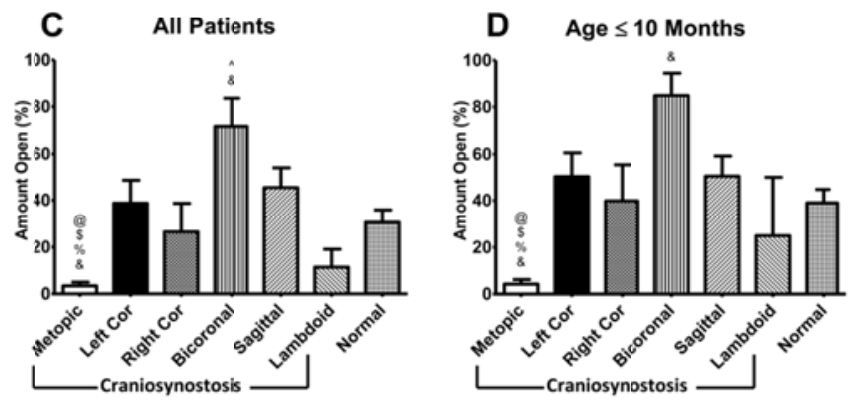

Figure 6. Fontanel and age dependency assessment. Age dependent affects were also observed in the different types of suture measurements calculated. An example of this was quantified when observing the percentage of the metopic suture that was open. When comparing a set of all patients (A) to just patients 10 months or younger (B), all mean values were higher in the younger patients because the sutures naturally close together with age. As they close together, the fontanels also begin to close. To remove the effects of the anterior fontanel, measurements were only taken from the outer three-fourths (segments 1-3) of the sutures (C-D) which showed a reduction in patency and an increase in significance between groups. For all graphs, significance of $P<.05$ for @ vs. Left Coronal, \# vs. Right Coronal, \$ vs. Bicoronal, \% vs. Sagittal, ^ vs. Lambdoid, \& vs. Normal.

\subsection{Age corrections}

The previously described method of dividing the suture into fourths and assessing the outer three-fourths, excluding the anterior fontanel, removed a source of error in quantifying the current state of the suture. The percentage open of the full 
metopic suture (see Figure 6A) displayed an elevated patency of $16.5 \%$ for metopic synostosis in comparison to the $3.4 \%$ when using segments 1-3 (see Figure 6C) which are the outer three-fourths of the suture path thereby excluding the anterior fontanel. This resulted in a $13 \%$ reduction in metopic synostosis when the fontanel was removed while most other groups maintained similar values. When patients older than 10 months were excluded (see Figure 6, B-D), the mean of each group increased due to a removal of older patients who have sutures that are naturally more closed at their age. While the standard error was not seen to decrease, this can be substantially attributed to the reduction in the number of patients analyzed. This illustrated the variation present when assessing patients of all different ages and two methods for reducing these effects by excluding the fontanels and comparing groups of similar ages when possible.

\subsection{Logistic regression predictive validation}

A multinomial logistic regression was trained to fit the entire patient dataset using the selected principal components that captured the variance in each measurement as it relates to distinguishing synostosis types. This produced an optimal set of equations:

$$
\begin{aligned}
\ln \frac{P(Y=1)}{P(Y=7)}=- & 3.459+1.494 x_{1}-3.758 x_{2}-1.092 x_{3}+0.411 x_{4}+0.123 x_{5}-0.743 x_{6}+2.137 x_{7}-9.803 x_{8}+ \\
& 4.983 x_{9}+22.999 x_{10}+429550.271 x_{11} \\
\ln \frac{P(Y=2)}{P(Y=7)}=- & 3.255-1.670 x_{1}-3.422 x_{2}-0.836 x_{3}+0.339 x_{4}+2.383 x_{5}+1.816 x_{6}-1.396 x_{7}+0.819 x_{8}- \\
& 4.302 x_{9}+23.847 x_{10}-171346.773 x_{11} \\
\ln \frac{P(Y=3)}{P(Y=7)}=- & 3.928+0.714 x_{1}-3.144 x_{2}-1.100 x_{3}-2.311 x_{4}+0.960 x_{5}+0.468 x_{6}+0.897 x_{7}-2.171 x_{8}+ \\
& 12.576 x_{9}+4.133 x_{10}+67296.221 x_{11} \\
\ln \frac{P(Y=4)}{P(Y=7)}=- & 4.239-0.278 x_{1}-2.350 x_{2}+2.069 x_{3}+1.091 x_{4}+0.511 x_{5}-0.948 x_{6}+0.445 x_{7}+0.037 x_{8}+ \\
& 6.765 x_{9}+18.727 x_{10}-127057.635 x_{11} \\
\ln \frac{P(Y=5)}{P(Y=7)}=- & 4.507+0.395 x_{1}-3.136 x_{2}+1.777 x_{3}-1.517 x_{4}+0.077 x_{5}-1.065 x_{6}-0.967 x_{7}-1.953 x_{8}+ \\
& 0.037 x_{9}+17.188 x_{10}-172990.142 x_{11} \\
\ln \frac{P(Y=6)}{P(Y=7)}= & -5.869-0.802 x_{1}-3.395 x_{2}-0.709 x_{3}+0.611 x_{4}+0.617 x_{5}-2.851 x_{6}+0.894 x_{7}-15.329 x_{8}+ \\
& 27.247 x_{9}+19.650 x_{10}-890362.037 x_{11}
\end{aligned}
$$

Each of these equations was set equal to a probability ratio of different outcomes with each outcome (Y) numbered 1 through 6 corresponding to a type of synostosis with 7 being the normal patient group, used as a baseline. That way each of these equations was trained by comparing the type of synostosis to the normal measurements. The probabilities resulting from these equations were found by plugging in the specific principal components for $\mathrm{x}_{1}$ to $\mathrm{x}_{11}$ and using the fact that the sum of the probabilities equals one as the seventh equation. It is difficult to determine the importance of each principal component from the beta coefficients alone because the components are scaled values based on the measurements they incorporate, however, the relative scaling and sign changes between equations provide some information about their effect. Besides this information, eigenvectors calculated by PCA revealed the variables with the most significant contribution to each principal component. Table 4 contains the absolute value of the eigenvector components corresponding with each of the 35 variables on the left. The 11 principal components shown were determined by feature selection from the original 35 to be the most predictive of synostosis type. Since higher magnitude signifies a more significant contribution to the corresponding component, the values greater than 0.2 are highlighted in yellow to display the most significant variables incorporated in each component. 
Table 4. Significant contributions to each principal component (greater than 0.2 in bold)

\begin{tabular}{|c|c|c|c|c|c|c|c|c|c|c|c|}
\hline & \multicolumn{11}{|c|}{ Principal components } \\
\hline & 1 & 2 & 3 & 4 & 5 & 6 & 7 & 8 & 9 & 10 & 11 \\
\hline Sex & 0.077 & 0.013 & 0.022 & 0.052 & 0.083 & 0.359 & 0.017 & 0.008 & 0.006 & 0.004 & 0.000 \\
\hline Age & 0.052 & 0.030 & 0.015 & 0.039 & 0.045 & 0.112 & 0.235 & 0.082 & 0.012 & 0.018 & 0.000 \\
\hline \multicolumn{12}{|l|}{ Volume Ratios } \\
\hline Right $\%$ & 0.038 & 0.075 & 0.309 & 0.335 & 0.042 & 0.108 & 0.100 & 0.034 & 0.005 & 0.002 & 0.407 \\
\hline Left $\%$ & 0.039 & 0.074 & 0.309 & 0.335 & 0.042 & 0.107 & 0.101 & 0.031 & 0.002 & 0.003 & 0.408 \\
\hline Front $\%$ & 0.348 & 0.107 & 0.086 & 0.084 & 0.124 & 0.085 & 0.022 & 0.006 & 0.012 & 0.201 & 0.377 \\
\hline Back \% & 0.349 & 0.107 & 0.085 & 0.083 & 0.125 & 0.084 & 0.021 & 0.004 & 0.010 & 0.186 & 0.376 \\
\hline Front Left $\%$ & 0.334 & 0.067 & 0.190 & 0.043 & 0.102 & 0.105 & 0.019 & 0.028 & 0.106 & 0.188 & 0.194 \\
\hline Front Right \% & 0.314 & 0.130 & 0.020 & 0.189 & 0.128 & 0.055 & 0.056 & 0.037 & 0.080 & 0.193 & 0.212 \\
\hline Back Left \% & 0.128 & 0.113 & 0.235 & 0.337 & 0.098 & 0.062 & 0.098 & 0.019 & 0.055 & 0.089 & 0.380 \\
\hline Back Right $\%$ & 0.207 & 0.008 & 0.306 & 0.243 & 0.025 & 0.139 & 0.073 & 0.016 & 0.038 & 0.091 & 0.397 \\
\hline A to P Ratio & 0.347 & 0.111 & 0.088 & 0.076 & 0.128 & 0.079 & 0.013 & 0.011 & 0.060 & 0.826 & 0.000 \\
\hline \multicolumn{12}{|l|}{ Metopic } \\
\hline Horizontal Dist. & 0.041 & 0.072 & 0.184 & 0.171 & 0.213 & 0.206 & 0.102 & 0.037 & 0.345 & 0.076 & 0.000 \\
\hline Average Vol. & 0.010 & 0.105 & 0.122 & 0.109 & 0.140 & 0.011 & 0.289 & 0.107 & 0.136 & 0.139 & 0.000 \\
\hline Total Vol. & 0.052 & 0.119 & 0.120 & 0.074 & 0.173 & 0.078 & 0.345 & 0.287 & 0.201 & 0.022 & 0.000 \\
\hline$\%$ Open & 0.066 & 0.054 & 0.072 & 0.137 & 0.081 & 0.171 & 0.173 & 0.114 & 0.150 & 0.129 & 0.000 \\
\hline Tangent Dist. Seg. & 0.036 & 0.074 & 0.215 & 0.154 & 0.112 & 0.271 & 0.242 & 0.051 & 0.333 & 0.075 & 0.000 \\
\hline Average Vol. Seg. & 0.006 & 0.120 & 0.128 & 0.106 & 0.113 & 0.056 & 0.251 & 0.108 & 0.069 & 0.127 & 0.000 \\
\hline \% Open Seg. & 0.075 & 0.054 & 0.094 & 0.141 & 0.047 & 0.187 & 0.083 & 0.106 & 0.166 & 0.106 & 0.000 \\
\hline \multicolumn{12}{|l|}{ Sagittal } \\
\hline Tangent Dist. & 0.079 & 0.209 & 0.130 & 0.133 & 0.113 & 0.053 & 0.131 & 0.062 & 0.056 & 0.071 & 0.000 \\
\hline Average Vol. & 0.199 & 0.128 & 0.114 & 0.061 & 0.024 & 0.268 & 0.190 & 0.060 & 0.005 & 0.030 & 0.000 \\
\hline Total Vol. & 0.169 & 0.147 & 0.125 & 0.059 & 0.019 & 0.346 & 0.229 & 0.063 & 0.021 & 0.011 & 0.000 \\
\hline Tangent Dist. Seg. & 0.087 & 0.188 & 0.180 & 0.144 & 0.045 & 0.022 & 0.159 & 0.023 & 0.045 & 0.137 & 0.000 \\
\hline Horizontal Dist. Seg. & 0.089 & 0.201 & 0.163 & 0.154 & 0.070 & 0.030 & 0.142 & 0.067 & 0.070 & 0.093 & 0.000 \\
\hline Average Vol. Seg. & 0.148 & 0.159 & 0.132 & 0.058 & 0.037 & 0.265 & 0.216 & 0.129 & 0.068 & 0.026 & 0.000 \\
\hline$\%$ Open Seg. & 0.189 & 0.143 & 0.010 & 0.052 & 0.037 & 0.219 & 0.056 & 0.174 & 0.028 & 0.017 & 0.000 \\
\hline \multicolumn{12}{|l|}{ Left Coronal } \\
\hline Tangent Dist. & 0.090 & 0.106 & 0.128 & 0.162 & 0.199 & 0.007 & 0.006 & 0.101 & 0.123 & 0.006 & 0.000 \\
\hline Average Vol. & 0.106 & 0.146 & 0.022 & 0.226 & 0.003 & 0.108 & 0.110 & 0.280 & 0.191 & 0.033 & 0.000 \\
\hline Tangent Dist. Seg. & 0.124 & 0.075 & 0.037 & 0.191 & 0.080 & 0.146 & 0.310 & 0.149 & 0.089 & 0.012 & 0.000 \\
\hline Horizontal Dist. Seg. & 0.154 & 0.106 & 0.026 & 0.160 & 0.126 & 0.108 & 0.207 & 0.198 & 0.005 & 0.009 & 0.000 \\
\hline Average Vol. Seg. & 0.082 & 0.160 & 0.008 & 0.192 & 0.063 & 0.140 & 0.121 & 0.107 & 0.212 & 0.041 & 0.000 \\
\hline Total Vol. Seg. & 0.042 & 0.163 & 0.000 & 0.273 & 0.043 & 0.170 & 0.073 & 0.024 & 0.034 & 0.007 & 0.000 \\
\hline \% Open Seg. & 0.114 & 0.058 & 0.057 & 0.247 & 0.019 & 0.031 & 0.085 & 0.287 & 0.053 & 0.006 & 0.000 \\
\hline \multicolumn{12}{|l|}{ Right Coronal } \\
\hline Tangent Dist. & 0.068 & 0.077 & 0.211 & 0.041 & 0.225 & 0.216 & 0.120 & 0.155 & 0.152 & 0.011 & 0.000 \\
\hline Horizontal Dist. & 0.091 & 0.080 & 0.198 & 0.025 & 0.274 & 0.245 & 0.194 & 0.125 & 0.211 & 0.005 & 0.000 \\
\hline Average Vol. & 0.087 & 0.221 & 0.147 & 0.083 & 0.038 & 0.003 & 0.002 & 0.078 & 0.333 & 0.001 & 0.000 \\
\hline Total Vol. & 0.081 & 0.224 & 0.148 & 0.074 & 0.023 & 0.004 & 0.010 & 0.080 & 0.153 & 0.001 & 0.000 \\
\hline$\%$ Open & 0.071 & 0.048 & 0.246 & 0.057 & 0.001 & 0.115 & 0.085 & 0.085 & 0.094 & 0.130 & 0.000 \\
\hline Tangent Dist. Seg. & 0.125 & 0.067 & 0.166 & 0.049 & 0.037 & 0.075 & 0.146 & 0.071 & 0.048 & 0.003 & 0.000 \\
\hline Horizontal Dist. Seg. & 0.131 & 0.084 & 0.170 & 0.040 & 0.100 & 0.111 & 0.062 & 0.027 & 0.056 & 0.013 & 0.000 \\
\hline Average Vol. Seg. & 0.062 & 0.234 & 0.091 & 0.080 & 0.084 & 0.067 & 0.017 & 0.221 & 0.172 & 0.007 & 0.000 \\
\hline$\%$ Open Seg. & 0.094 & 0.037 & 0.210 & 0.038 & 0.034 & 0.093 & 0.019 & 0.151 & 0.076 & 0.111 & 0.000 \\
\hline \multicolumn{12}{|l|}{ Lambdoid } \\
\hline Tangent Dist. & 0.012 & 0.146 & 0.005 & 0.054 & 0.331 & 0.047 & 0.032 & 0.029 & 0.029 & 0.020 & 0.000 \\
\hline Horizontal Dist. & 0.048 & 0.195 & 0.009 & 0.001 & 0.340 & 0.070 & 0.042 & 0.045 & 0.039 & 0.079 & 0.000 \\
\hline Average Vol. & 0.111 & 0.234 & 0.075 & 0.000 & 0.072 & 0.113 & 0.109 & 0.031 & 0.348 & 0.017 & 0.000 \\
\hline Total Vol. & 0.067 & 0.212 & 0.084 & 0.012 & 0.085 & 0.003 & 0.061 & 0.374 & 0.123 & 0.002 & 0.000 \\
\hline$\%$ Open & 0.049 & 0.217 & 0.025 & 0.019 & 0.315 & 0.045 & 0.124 & 0.215 & 0.150 & 0.038 & 0.000 \\
\hline Horizontal Dist. Seg. & 0.073 & 0.224 & 0.013 & 0.012 & 0.320 & 0.017 & 0.070 & 0.177 & 0.075 & 0.049 & 0.000 \\
\hline Average Vol. Seg. & 0.062 & 0.253 & 0.075 & 0.017 & 0.093 & 0.074 & 0.072 & 0.434 & 0.269 & 0.010 & 0.000 \\
\hline \% Open Seg. & 0.066 & 0.229 & 0.029 & 0.007 & 0.297 & 0.027 & 0.161 & 0.029 & 0.118 & 0.031 & 0.000 \\
\hline
\end{tabular}

Note. Left and right correspond to the patient's left and right side of the body. A to P ratio is the Anterior to Posterior Ratio. Seg. represents measurements done on only a segment of the suture (in this case, the outer three-quarters of the suture).

Each principal component was seen to have a few top contributing variables. Number 1 incorporated information from the intracranial volume measurement especially related to the volume distribution between the front and back of the skull. The 
second component was comprised mostly of the lambdoid suture measurements with a combination of some sagittal and right coronal suture measurements as well. And so on with the highest values in the columns corresponding to the most significant contributors.

Table 5. Logistic regression fit, characterization, and cross-validation

\begin{tabular}{|c|c|c|c|c|c|c|c|c|c|}
\hline \multicolumn{4}{|l|}{ Pseudo $\mathbf{R}^{2}$} & \multicolumn{3}{|c|}{ Full model characteristics } & \multicolumn{3}{|c|}{ Cross-validation results } \\
\hline McFadden & $\begin{array}{l}\text { McFadden } \\
\text { adjusted }\end{array}$ & Cox-snell & Nagelkerke & Accuracy & Mean Prob. & Deviance & $\begin{array}{l}\text { Error } \\
\text { rate }\end{array}$ & Accuracy & Mean Prob. \\
\hline 0.9816 & 0.9525 & 0.9687 & 0.9980 & 1 & 0.9876 & 7.5983 & 0.1314 & 0.8686 & 0.8440 \\
\hline
\end{tabular}

Four different pseudo $\mathrm{R}^{2}$ assessments (McFadden, McFadden Adjusted, Cox-Snell, and Nagelkerke) were conducted to analyze the data fitting, and all produced values over 0.95 indicating a good fit to the training data (see Table 5). It succeeded in predicting synostosis type for each patient with $100 \%$ accuracy and a mean probability or confidence in the prediction of 0.99 . The 10,000 fold cross-validation was done to assess the predictive accuracy using a different training set from validation set. This resulted in $86.9 \%$ average accuracy and a mean probability of 0.84 . Sensitivity analysis of the model showed the effect of the ill conditioned lambdoid prediction and increased average accuracy to $91.9 \%$ when the lambdoid patients were not considered and the model was trained for the other six outcomes.

\section{Discussions}

The image processing algorithm developed for craniosynostosis quantification succeeded in characterizing the current state of the developing cranium as verified by the successful prediction of synostosis type using logistic regression predictive modeling. This algorithm allowed the quantification and processing of a CT scan for intracranial volume asymmetries and cranial suture measurements not previously possible. This quantification enabled the characterization of previously observed effects caused by premature suture fusions.

\subsection{Validation}

The suture measurements and snake algorithm's refined bone boundaries were first successfully validated using correlation plots comparing measurement values from each border to one another. Since the measurements would result in the same value when the borders are identical, each data point should have been close to the $\mathrm{y}=\mathrm{x}$ line to establish a correlation. While some measurements were not well validated by the manually drawn borders, these effects were compensated for in the quantification algorithm and exclusion of specific features. A few outlying validation data points drastically reduced the $\mathrm{R}^{2}$ values observed in the sagittal suture validation. These outliers were due to a random selection of two planes located in the anterior fontanel. The extreme bone width of the fontanel in very young patients was seen to produce an automatic determination of a fused suture when little or no bone was present in the $50 \mathrm{~mm} \times 50 \mathrm{~mm}$ window. However, these situations did not pose a problem for the quantification algorithm because the anterior fontanel was excluded from our measurements automatically. With the outliers accounted for using the three-fourths modification, there was a dramatic increase in the $\mathrm{R}^{2}$ value for the sagittal suture measurements. For the tangent and horizontal distances in the sagittal suture, a removal of these two outliers resulted in new $\mathrm{R}^{2}$ values of 0.97 and 0.93 respectively. For the percentage open of the sagittal suture, the one outlier than included an average of these planes was excluded to produce a new $\mathrm{R}^{2}$ value of 0.65 . With these corrections that had already been accounted for in the algorithm, this resulted in a successful validation of the distance measurements, the bone volume, and the percentage open of the sutures on the automatically determined boundary.

In evaluating the accuracy of the lambdoid sutures measurements, it became apparent that at least a five-fold resolution difference exists in the CT scans in the vertical direction (slice separation) compared to the higher quality horizontal image slice (pixel separation). This resulted in a less definitive border in the reconstructed images usually on the top and bottom 
of the bone, not affecting the distance or percentage open measurements but having some effect on the bone volume. This was true for sutures located on the top of the skull, but since the lambdoid suture is positioned farther back on the skull where the bone junction is more vertical than the rest, the suture boundaries were affected making it more difficult to define a precise bone boundary, which resulted in differences in manual and automatic boundaries seen in the validation.

This ambiguity in the vertical border of the other sutures resulted in a larger variation in the manual and automatic borders causing the bone height to be excluded from further analysis. The bone volume also experienced some variation due to this as well, but not near enough to warrant exclusion. The other three measurements were not affected by variations in the vertical border as they assess the distance between the two bones of the suture to see if there is a separation present.

The percentage open had less data points as each was calculated by averaging the value from each of the five images in the suture since a value of one or zero was determined per slice. While there was some variation seen in the accuracy of this measurement, it was more difficult to assess from a small scale representation since it is calculated on the assumption that the larger overall sample size will compensate for minimal classification errors with image sets typically containing between 100 and 250 reconstructed images.

\subsection{Total I CV and ratios}

Craniosynostosis has been previously observed to alter the intracranial volume distribution as cranial development continues despite the constriction ${ }^{[4,21]}$. The developing cranium continually expands to compensate for rapid cognitive development in the first few years after birth. The maintained total intracranial volume measured by the program quantified this effect as the normal patient group formed a growth curve similar to that of the craniosynostosis patients. A few patients fell outside of this relative range, but these were patients that suffered from more severe constriction. This was observed in cases of bilateral constriction typically associated with bicoronal synostosis or cases of delayed surgical intervention which allows the deformation to further progress. This intracranial volume growth curve was similar to those constructed previously on sets of normal patients ${ }^{[22,23]}$. Since the majority of craniosynostosis and normal patients fell into this standard range, this alluded to the fact that the cranial expansion was still occurring even when one of the sutures fuses prematurely. This effect was also quantified by fitting a non-linear regression to all craniosynostosis cases and one to all normal cases. Their overlapping confidence intervals verify the observed affect.

From this, it was observed that a reduction in total intracranial volume was not seen at the time of surgery, but the distortion in cranial expansion was present. Therefore, it was beneficial to conduct an asymmetry analysis of the total intracranial volume using the suture boundaries to determine the lines of symmetry. From these divisions, the importance of the volume percentages in each quadrant and the volume ratios were identified. These measurements allowed for asymmetric quantification and demonstrated the observed phenomena of a premature suture fusion's effect on calvarial expansion to compensate for brain growth. Besides the quadrant volume increases, the ratio of anterior to posterior volume showed separation between cases of metopic and sagittal synostosis because of increased constriction on the anterior portion on the calvaria in metopic synostosis versus sagittal synostosis that causes an elongation of the skull resulting in an increased volume anterior to the coronal suture. The other volume measurements calculated did not produce as noticeable of a separation between synostosis types, but the conversion to the percentages of the total intracranial volume was successful for normalizing measurements across the age range and increasing separation.

\subsection{Suture measurements}

Similar to the observed intracranial volume compensation, the effects of suture fusion were not localized in more severe cases. A single suture synostosis often affected other sutures as well due to the displaced volume. This volume compensation seen often resulted in a wider gap in the sutures present in the region of compensation especially when a patient is younger and their sutures are less developed. This effect was prominently portrayed in the case of bicoronal synostosis where there was more cranial restriction at a young age resulting in a wide open midline of the skull including 
the metopic and sagittal sutures. This same effect was less exaggerated but present in sagittal synostosis where the elongation of the skull results in more wide open left and right coronal sutures.

Some of the more minor measurement differences can be attributed to the anatomical changes with age and variations between the different synostosis groups especially observed in the metopic suture that has an earlier natural closure time around 9 months ${ }^{[24]}$. The average age of bicoronal patients (4.9 months) was lower than normal patients (7.7 months) since bicoronal synostosis often requires earlier surgical intervention due to the more debilitating constriction present. The reduction of patency in cases of lambdoid synostosis can be attributed to the older average age of the patients (12.6 months), but no significant differences were seen due, in part, to the limited number of patients with lambdoid synostosis in this study due to its low incidence.

The bone volume and percentage open quantified what was occurring in each suture in cases of craniosynostosis and had inversely proportional relationships to one another. The tangent and horizontal distances produced similar results to the percentage open measurements with a better quantification of the width of the bone separation, and the average bone volume in each reconstructed image was similar to total bone volume results.

The comparison to the normal patient group was not as useful in the bone volume measurements because the normal CT scans had a $5 \mathrm{~mm}$ separation between image slices (twice that of the craniosynostosis scans) and therefore, used twice the vertical approximation of the craniosynostosis scans resulting in an increased over-approximation of the bone size. As stated in the validation, vertical quality was not important in the distance and percentage open measurements, but bone volume utilized the vertical borders in its quantification.

\subsection{Age corrections}

Another prominent effect observed was the role that age differences played when assessing the suture measurements. Some age effects were mitigated by using only the outer three-fourths subsets of the suture for measurements, however, the distance between the bones rapidly decreases in the first two years making any bone measurements at the sutures still have some age-dependent affect. The metopic suture also complicated the quantification because it has been shown to have an even quicker normal closure that begins three months after birth and is typically completely closed after nine to twelve months ${ }^{[24]}$. The remaining cranial sutures normally narrow dramatically within the first two years after birth, but do not have full bone fusion for 30 to 40 years ${ }^{[4,5]}$.

Calculating intracranial volume asymmetries using quadrant volume distribution and measuring suture characteristics from 3D reconstructions were two effective techniques that have not been described previously to quantify the process of growth in the developing skull. These measurements allowed for a better understanding of the patient set beyond basic age comparisons and visual deformity to evaluate a patient's condition and allowed for comparisons between patients. Better understanding and assessment of the current progression of the disease can lead to better treatment planning and outcomes for patients.

\subsection{Predictive validation}

The image processing algorithm implemented for craniosynostosis quantification was validated by the successful prediction of synostosis type using logistic regression. The quantification of previous craniosynostosis observations further validates measurements produced from this program. These measurements combined with the multinomial logistic regression's correct classification of each patient's synostosis type with $100 \%$ accuracy and fitting of the data with pseudo $\mathrm{R}^{2}$ values greater than 0.95 represent a well-trained model. The stability of the model was further assessed using 10,000 fold cross-validation which resulted in an average accuracy of $86.9 \%$ and a mean probability of 0.84 . These results show a sufficient accuracy for a seven class model with a probability term that expresses confidence in each prediction. Some of the error in classification can be attributed to a low number of patients associated with specific outcomes such as lambdoid and bicoronal synostosis. It is difficult to guarantee prediction accuracy when the model may only be trained on a handful 
of patients for a specific synostosis type which is the case in cross-validation where each synostosis group is broken into smaller groups for training and validation. Therefore, sensitivity analysis that yielded a $91.9 \%$ average accuracy with the removal of the lambdoid patient group helped reveal one main source of error caused by the limited incidence of certain types of synostosis.

These techniques can be extrapolated to quantifying the cranial state from any CT scan. Therefore, they are also methods that can be used for an assessment of surgical procedures and complications. This methodology of cranial quantification and prediction can also be used for future prediction of surgical complications including re-synostosis when used in conjunction with surgical information and patient history on a large enough scale. It can then provide insight into surgical planning and operation outcomes by providing a better understanding of the state of the patient at the time of the surgery.

\section{Acknowledgements}

The authors would like to thank Meredith Capasse for her assistance collecting the CT scans and patient information. This work was funded by Children's Healthcare of Atlanta and US PHS Grant P50FDO-04193.

\section{References}

[1] Boulet SL, Rasmussen SA, Honein MA. A population-based study of craniosynostosis in metropolitan Atlanta, 1989-2003. Am J Med Genet A. 2008; 146A(8): 984-91. PMid:18344207. http://dx.doi.org/10.1002/ajmg.a.32208

[2] Hunter AG, Rudd NL. Craniosynostosis. I. Sagittal synostosis: its genetics and associated clinical findings in 214 patients who lacked involvement of the coronal suture(s). Teratology. 1976; 14(2): 185-93. PMid:982314. http://dx.doi.org/10.1002/tera.1420140209

[3] Lajeunie E, Le Merrer M, Bonaiti-Pellie C, et al. Genetic study of nonsyndromic coronal craniosynostosis. Am J Med Genet. 1995; 55(4): 500-4. PMid:7762595. http://dx.doi.org/10.1002/ajmg.1320550422

[4] Kirmi O, Lo SJ, Johnson D, et al. Craniosynostosis: A Radiological and Surgical Perspective. Seminars in Ultrasound, CT, and MRI. 2009; 30(6): 492-512. http://dx.doi.org/10.1053/j.sult.2009.08.002

[5] Kabbani H, Raghuveer TS. Craniosynostosis. Am Fam Physician. 2004; 69(12): 2863-70. Epub 2004/06/30. PMid:15222651.

[6] Wilkie AO. Craniosynostosis: genes and mechanisms. Hum Mol Genet. 1997; 6(10): 1647-56. PMid:9300656. http://dx.doi.org/10.1093/hmg/6.10.1647

[7] Foster KA, Frim DM, McKinnon M. Recurrence of synostosis following surgical repair of craniosynostosis. Plast Reconstr Surg. 2008; 121(3): 70e-6e. PMid:18317088. http://dx.doi.org/10.1097/01.prs.0000299393.36063.de

[8] Williams JK, Cohen SR, Burstein FD, et al. A longitudinal, statistical study of reoperation rates in craniosynostosis. Plast Reconstr Surg. 1997; 100(2): 305-10. PMid:9252595. http://dx.doi.org/10.1097/00006534-199708000-00003

[9] Esparza J, Hinojosa J. Complications in the surgical treatment of craniosynostosis and craniofacial syndromes: apropos of 306 transcranial procedures. Childs Nerv Syst. 2008; 24(12): 1421-30. PMid:18769932. http://dx.doi.org/10.1007/s00381-008-0691-8

[10] Esparza J, Hinojosa J, Garcia-Recuero I, et al. Surgical treatment of isolated and syndromic craniosynostosis. Results and complications in 283 consecutive cases. Neurocirugia (Astur). 2008; 19(6): 509-29.

http://dx.doi.org/10.1016/S1130-1473(08)70201-X

[11] Panchal J, Uttchin V. Management of craniosynostosis. Plast Reconstr Surg. 2003; 111(6): 2032-48; quiz 49. PMid:12711969. http://dx.doi.org/10.1097/01.PRS.0000056839.94034.47

[12] Sahin B, Acer N, Sonmez OF, et al. Comparison of four methods for the estimation of intracranial volume: a gold standard study. Clin Anat. 2007; 20(7): 766-73. PMid:17708568.

[13] Marcus JR, Domeshek LF, Das R, et al. Objective three-dimensional analysis of cranial morphology. Eplasty. 2008 ; 8: e20. PMid:18464892.

[14] de Oliveira ME, Hallila H, Ritvanen A, et al. Feature-invariant image registration method for quantification of surgical outcomes in patients with craniosynostosis: a preliminary study. J Pediatr Surg. 2011; 46(10): E1-8. PMid:22008355. http://dx.doi.org/10.1016/j.jpedsurg.2011.04.095

[15] Hermann CD, Richards MA, Olivares-Navarrete R, et al. Algorithm to assess cranial suture fusion with varying and discontinuous mineral density. Ann Biomed Eng. 2012; 40(7): 1597-609. PMid:22350663. http://dx.doi.org/10.1007/s10439-012-0520-9

[16] Vidakovic B. Statistics for bioengineering sciences: with MATLAB and WinBUGS support. New York: Springer. 2011. http://dx.doi.org/10.1007/978-1-4614-0394-4 
[17] Peduzzi P, Concato J, Kemper E, et al. A simulation study of the number of events per variable in logistic regression analysis. J Clin Epidemiol. 1996; 49(12): 1373-9. http://dx.doi.org/10.1016/S0895-4356(96)00236-3

[18] Hermann CD, Lawrence K, Olivares-Navarrete R, et al. Rapid re-synostosis following suturectomy in pediatric mice is age and location dependent. Bone. 2013; 53(1): 284-93. PMid:23201269. http://dx.doi.org/10.1016/j.bone.2012.11.019

[19] Duc G, Largo RH. Anterior fontanel: size and closure in term and preterm infants. Pediatrics. 1986; 78(5): 904-8. PMid:3763303.

[20] Behrman RE, Kliegman R, Nelson WE. Nelson textbook of pediatrics. 15th ed. Philadelphia: W.B. Saunders. 1996.

[21] Bristol RE, Lekovic GP, Rekate HL. The effects of craniosynostosis on the brain with respect to intracranial pressure. Semin Pediatr Neurol. 2004; 11(4): 262-7. PMid:15828710. http://dx.doi.org/10.1016/j.spen.2004.11.001

[22] Sgouros S, Goldin JH, Hockley AD, et al. Intracranial volume change in childhood. J Neurosurg. 1999; 91(4): 610-6. PMid:10507383. http://dx.doi.org/10.3171/jns.1999.91.4.0610

[23] Kamdar MR, Gomez RA, Ascherman JA. Intracranial volumes in a large series of healthy children. Plast Reconstr Surg. 2009; 124(6): 2072-5. PMid:19952664. http://dx.doi.org/10.1097/PRS.0b013e3181bcefc4

[24] Vu HL, Panchal J, Parker EE, et al. The timing of physiologic closure of the metopic suture: a review of 159 patients using reconstructed 3D CT scans of the craniofacial region. J Craniofac Surg. 2001; 12(6): 527-32.

http://dx.doi.org/10.1097/00001665-200111000-00005 